\section{Reflection of X-rays with a Change of Frequency}

A CHANGE of frequency in the scattering of light or of X-rays is a familiar phenomenon. On the other hand, the coherent $X$-ray reflections given by crystals are a consequence of classical optics and any change of frequency in such reflection might seem inadmissible. A closer consideration shows, however, that even on the classical theory, a change of frequency might accompany a coherent reflection, if the structure-amplitude of the crystal planes responsible for the reflection were to vary periodically with time. This would occur, for example, when the interpenetrating lattices in the crystal oscillate against each other with the characteristic infra-red frequencies and produce pulsations of the electron-density in the crystal. On the principles of the quantum theory, therefore, such lattice oscillations, though initially non-existent, may be excited as the result of the incident radiation and give rise to a coherent reflection of altered frequency.

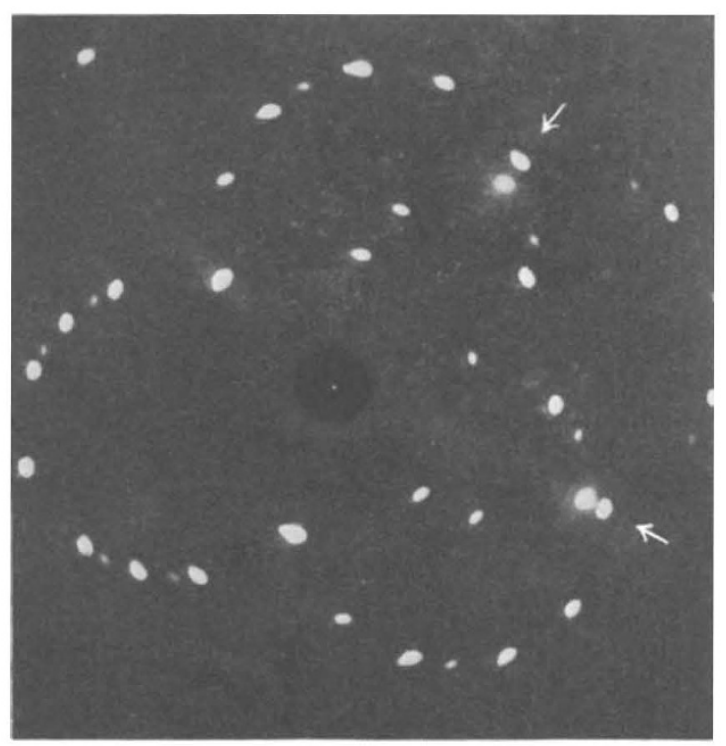

Laue Pattern of Sodium Nitrate.

Consider the effect on a beam of monochromatic $\mathrm{X}$-radiation passing through a crystal of a set of spacings having a large structure-amplitude. If the angle of incidence on these spacings is different from that given by the Bragg formula, the usual type of $\mathrm{X}$-ray reflection would be forbidden. A periodic variation with time of the structure-amplitude, if it has the same phase throughout the crystal, would also fail to give rise to a reflection with modified frequency. In other words, reflections with and without modification of frequency would occur only in accompaniment with each other. On the other hand, if the variation of structure-amplitude has not the same phase everywhere, the two types of reflection may occur independently. While the unmodified reflections could appear only at the correct Bragg angle of incidence on the crystal spacings, the modified reflections would not be restricted to such incidences. The direction in which they appear would correspond with that of geometric reflection, not from the crystal spacings, but from the plane waves of constant phase of the structure-amplitude variations. The inclination of these waves would, in fact, be uniquely determined by the circumstances of each reflection, as these must satisfy the Bragg condition as well. In other words, on the quantum theory, only that particular mode of oscillation would be excited which would give the observed reflection.

A crystal of sodium nitrate gives excellent examples of these modified reflections. The accompanying illustration is a Laue pattern obtained with such a crystal, the direction of the incident $\mathrm{X}$-ray pencil (from a copper target) being normal to one of the three pairs of the rhombohedral cleavage faces and therefore equally inclined to the other two pairs of such faces. Along the two radial lines marked in the figure will be seen following each other in order, the usual Laue reflection from the cleavage spacings and the modified reflections corresponding to the incident copper $K_{\alpha}$ and copper $K_{\beta}$ monochromatic radiations. Still nearer the centre will be seen the faint radial streaks corresponding to the modified reflections of the incident white radiations.

\section{V. Raman.}

P. NILAKantan.

Department of Physics,

Indian Institute of Science, Bangalore.

April 13.

\section{Fission of Uranium and Thorium under Deuteron Bombardment}

Is continuation of the experiments on uranium fission under deuteron bombardment reported by Gant $^{1}$ from this laboratory, we have carried out a chemical separation of the recoil products. The particles projected from the uranium target under deuteron bombardment were collected on an aluminium foil using the experimental arrangement described by Gant. The foil was then dissolved in aqua regia and, after adding the necessary carriers, platinum, barium and lanthanum separations were made in the usual way. The activities observed were $15 \mathrm{~min} ., \mathrm{l} \mathrm{hr}$. and 30-40 hr. with the platinum fraction, $11 \mathrm{~min}$. and $80 \mathrm{~min}$. with the barium fraction, and $4 \mathrm{hr}$. with the lanthanum fraction. Some of the periods are thus found to correspond to those obtained with neutron fission in uranium ${ }^{2}$, in agreement with Gant's conclusion that $9 \mathrm{Mev}$. deuterons are capable of producing fission in uranium. Control experiments with lead and bismuth targets failed to show any appreciable activities in the carriers.

Using the same experimental arrangement as before, we have bombarded thorium with 9 Mev. deuterons, a thin layer of metallic thorium being used as the target. Seven minutes after a $6 \mu$ amp. min. bombardment, an aluminium foil $F^{\prime}\left(3 \cdot 3 \mathrm{mgm} . / \mathrm{cm} .^{2}\right)$, which had been placed inside the side pocket of the target box and protected from the low-energy products due to mechanical disintegration of the target by $2 \mathrm{~mm}$. S.P. aluminium, gave an activity of about 1,200 counts per min., whereas a second aluminium foil $D$ which was placed behind the foil $F$ and therefore measured the scattered deuteron effect, gave an activity of 100 counts per min., decaying with the well-known $2.4 \mathrm{~min}$. period of ${ }^{28} \mathrm{Al}$. The activity produced in a third aluminium foil $N$, placed inside the pocket but separated from the foil $D$ by a copper sheet $\left(\frac{1}{2} \mathrm{~mm}\right.$. thick), was of the order of 20 counts 